\title{
miR-196b promotes lung cancer cell migration and invasion through the targeting of GATA6
}

\author{
HONGLI LI ${ }^{1}, \mathrm{CHAO} \mathrm{FENG}^{2}$ and SONGTAO $\mathrm{SHI}^{3}$ \\ Departments of ${ }^{1}$ Operation Room, ${ }^{2}$ Surgery and ${ }^{3}$ Thoracic Surgery, \\ Eastern Medical District of Linyi People's Hospital, Linyi, Shandong 276034, P.R. China \\ Received March 19, 2018; Accepted May 4, 2018
}

DOI: $10.3892 / \mathrm{ol} .2018 .8671$

\begin{abstract}
MicroRNAs (miRNAs) have been proven to regulate gene expression at the protein translation level. miRNA abnormal expression has been associated with the development of lung cancer. In the present study, we aimed to investigate the mechanism of miR-196 in non-small cell lung cancer (NSCLC). The miR-196b and GATA-6 (GATA6) expression levels were examined in NSCLC by RT-qPCR and western blot analysis. Transwell assay was used to assess cell migration and invasion. Moreover, the specific target of miR-196b in NSCLC was verified by the luciferase reporter assay. The expression of miR-196b was higher in both NSCLC tissues and cells than the normal levels. Specifically, the miR-196b mimic group in NSCLC cells markedly promoted cell migration and invasion, while the miR-196b inhibitor group exhibited the opposite effect. Furthermore, GATA6 was verified as a specific target of miR-196b in NSCLC cells and GATA6 could attenuate the migratory and invasive ability of NSCLC cells regulated by miR-196b. In addition, the relationship between GATA6 and miR-196b expression was negatively correlated in NSCLC tissues. Thus, miR-196b enhanced NSCLC cell migration and invasion via the downregulation of GATA6, indicating its potential application in NSCLC diagnosis and therapy.
\end{abstract}

\section{Introduction}

Lung cancer ranks among the most rapidly growing types of cancer with a high morbidity and mortality rate. Also, it is one of the most serious malignancies for population health and life $(1,2)$. Non-small cell lung cancer (NSCLC) accounts for $70-80 \%$ among all types of lung cancer and the survival rate is approximately $11 \%$ (3). Patient relapse may lead to disease metastasis and consequently, death. Therefore, identification of novel treatments for human NSCLC is required.

Correspondence to: Dr Songtao Shi, Department of Thoracic Surgery, Eastern Medical District of Linyi People's Hospital, 233 Fenghuang Street, Hedong, Linyi, Shandong 276034, P.R. China E-mail: t2c959@163.com

Key words: miR-196b, migration, invasion, NSCLC, GATA6
MicroRNAs (miRNAs), endogenous non-coding singlestranded RNAs bound to the 3'-untranslated region (3'-UTR) of mRNAs lead to the proliferation, metastasis and invasion of cancer (4-6). Previous studies have shown that miR-196 has an aberrant expression in various tumors and has been involved in the progression of cancers. For example, Mueller and Bosserhoff showed that silencing miR-196a may deregulate several genes in melanoma cells ito nfluence melanoma progression (7). miR-196a has been found to be highly-expressed in colorectal cancer cells and it may enhance cancer cell migration and invasion (8). In addition, a study showed that the re-expression of miR-196b significantly contributed to leukemia development (9). The effect of miR-196 on the malignant progression of gliomas has also been demonstrated (10). Moreover, miR-196s acted as potent suppressors in the cell migration and metastasis of breast tumors (11). It has been previously reported that miR-196b is significantly upregulated in lung cancer and is considered to be a potential marker of survival (12). However, the underlying mechanism of miR-196b in the NSCLC cell process has yet to be explored.

Transcription factor GATA-6 (GATA6) has been identified to be involved in the development, proliferation, and differentiation of several organs $(13,14)$. GATA6 promoted cholangiocarcinoma cell invasion and metastasis and acted as a potential oncogene in cholangiocarcinoma $(15,16)$. GATA6 has been shown to be misregulated in colon cancer cells, suggesting a relevant role in the progression of colon cancer $(17,18)$. Moreover, GATA6 was verified to inhibit lung adenocarcinoma metastasis (19).

In our study, we showed the upregulation of miR-196b and downregulation of the GATA6 expression in NSCLC. GATA6 has been characterised as a direct target gene of miR-196b in NSCLC. The relationship between miR-196b and GATA6 protein level was found to be negatively correlated in NSCLC tissues. Overall, we drew a conclusion that miR-196b may enhance NSCLC cell migration and invasion by targeting GATA6.

\section{Materials and methods}

Tissue sample collection. NSCLC tissues and normal lung tissues were obtained from 40 paired patients all of whom signed written informed consent after surgery at The Eastern 
Medical District of Linyi People's Hospital (Linyi, China). Then, we stored the samples in a $-80^{\circ} \mathrm{C}$ refrigerator. All the experiments were approved by the Ethics Committee of The Eastern Medical District of Linyi People's Hospital (Linyi, China).

Cell culture. The NSCLC cell lines (A549, H226, H1650, H1299, SPC-A1) were purchased from the American Type Culture Collection (ATCC, Manassas, VA, USA). The cells were cultured in RPMI-1640 medium (Gibco; Thermo Fisher Scientific, Inc., Carlsbad, CA, USA) containing 10\% fetal bovine serum, penicillin $(100 \mathrm{U} / \mathrm{ml})$ and streptomycin $(100 \mathrm{U} /$ $\mathrm{ml}$ ), which was incubated at $37^{\circ} \mathrm{C}$ in a $5 \% \mathrm{CO}_{2}$ environment.

Transfection of miR-196b. miR-196b mimic, miR-196b inhibitor, control mimic and control inhibitor were transfected to A549 cells or H226 cells using Lipofectamine 2000 (Invitrogen; Thermo Fisher Scientific, Inc.) the next day when the cells were $70-80 \%$ confluent.

Overexpression of GATA6. The GATA6 plasmid was purchased from GeneCopoeia (Germantown, MD, USA), and transfected in A549 cells using Lipofectamine 2000 (Invitrogen; Thermo Fisher Scientific, Inc.).

Western blot analysis. After transfection for $48 \mathrm{~h}$, RIPA lysis containing proteinase inhibitors (Beyotime Institute of Biotechnology, Haimen, China) was used to extract total protein. The protein concentrations were tested with the BCA kit (Beyotime Institute of Biotechnology). The total proteins $(50 \mu \mathrm{g})$ were added into the well with SDS-PAGE and electrophoresis at $60 \mathrm{~V}$ was performed when bromophenol blue ran out of the bottom. The proteins were then transferred to nitrocellulose filter (NC) membranes, and skimmed milk (5-10\%) was used to block he membranes at room temperature for $2 \mathrm{~h}$. Subsequently, primary antibodies (GATA6, Abcam, Cambridge, UK; GAPDH, Cell Signaling Technology, Inc., Danvers, MA, USA) were added in to incubate the samples at $4^{\circ} \mathrm{C}$ overnight. After being washed with 1X TBST (pH 7.4) three times, the secondary antibodies were added in and incubated at room temperature for $2 \mathrm{~h}$. Protein bands were detected using the chemiluminescence method (ECL; Millipore Billerica, MA, USA). GAPDH served as a loading control.

RNA isolation and RT-qPCR. We used TRIzol Reagent (Invitrogen; Thermo Fisher Scientific, Inc.) to extract total RNA from the cell lines or tissue samples (Invitrogen; Thermo Fisher Scientific, Inc.), then, we used the BioPhotometer (Eppendorf, Hamburg, Germany) to determine the concentration of the total RNA. All-in-One ${ }^{\mathrm{TM}}$ miRNA First-Strand cDNA synthesis kit was used to synthesize cDNA, and RT-qPCR was performed using the TaqMan PCR probes. The of the primer sequences were as follows: miR-196b-F: TAG GTACCACTTTATCCCGTTCACCA, miR-196b-R: ATC TCGAGGCAGGGAGAGAGGAATAA; GATA6-F: CTC CAACTTCCACCTCTTCTAAC, GATA6-R: TGGTGTGGT GGAGTCG; U6-F: GCCCATCTTGACCCGAAT, U6-R: AACGCTTCACGAATTTGCGT; GAPDH-F: ACAGTCAG CCGCATCTTCTT, GAPDH-R: ACGACCAAATCCGT
TGACTC. GAPDH and U6 were used as endogenous controls. Relative expression levels of genes analysed were calculated using the $2^{-\Delta \Delta C q}$ method.

Cell migration assay. A549 and H226 cell migration was measured using a Transwell chamber with a polycarbonic membrane with $8-\mathrm{mm}$ pore size in vitro. NSCLC cells were treated with different transfection for $24 \mathrm{~h}$. Then, $1 \times 10^{5}$ cells were seeded into the upper chamber and a complete culture medium with $20 \%$ FBS was added to the lower chamber as an attractant. After incubation for $24 \mathrm{~h}$ at $37^{\circ} \mathrm{C}$, in a $5 \% \mathrm{CO}_{2}$ environment, the media were removed. The cotton swabs were used to remove the cells from the top chamber. Moreover, the $100 \%$ methanol was used to fix the cells migrated to the lower membrane and $0.1 \%$ crystal violet was used to stain the migrating cells. The number of cells was quantified by a microscope (Olympus, Tokyo, Japan).

Cell invasion assay. A549 and H226 cells invasion was measured using a millicell invasion chamber with $8-\mu \mathrm{m}$ pore size polycarbonate membranes (Neuro Probe Inc., Gaithersburg, MD, USA) pre-coated with $2 \mathrm{mg} / \mathrm{ml}$ Matrigel (BD Bioscience, San Jose, CA, USA). Then $1 \times 10^{5}$ cells were added to the upper chamber coated with Matrigel and a complete culture medium with $20 \%$ FBS was added to the lower chamber as an attractant and invaded for $24 \mathrm{~h}$. The cotton swabs were used to remove the cells in the top chamber. Moreover, $100 \%$ methanol was used to fix the invaded cells to the lower membrane and $0.1 \%$ crystal violet was used to stain the invading cells. The number of cells was quantified by microscope (Olympus).

Dual luciferase reporter assay. The wild-type and mutated miR-196b putative targets on GATA6 3'UTR were cloned into pGL3-promoter vector, a final concentration of $100 \mathrm{nM}$ of cells of different transfection treatments were collected $48 \mathrm{~h}$ after transfection using Lipofectamine 2000, and luciferase activity was assayed with the dual-luciferase reporter assay system (Promega, Madison, WI, USA).

Statistical analysis. The experiments were repeated three times. SPSS v.19.0 software (IBM Corp., Armonk, NY, USA) was used to perform the statistical analyses as well as GraphPad Prism 5.02 software (GraphPad Software, Inc., La Jolla, CA, USA) to complete graph presentations. All data were presented as mean \pm SD. Student's t-test or post hoc test after one-way analysis of variance (ANOVA) in SPSS were used to analyze the differences between the groups. $\mathrm{P}<0.05$ was considered to indicate a statistically significant difference.

\section{Results}

Detection of miR-196b mRNA and GATA6 protein expression in NSCLC tissues. First, we investigated the miR-196b expression in eight NSCLC tissue specimens and their corresponding non-cancerous tissue (NCT) specimens using RT-qPCR. The results showed that the miR-196b expression was markedly higher in NSCLC samples (Fig. 1A). The role of miRNA via targeting the 3'-UTR of mRNA is involved in the regulation of gene expression. Then, we used the TargetScan 


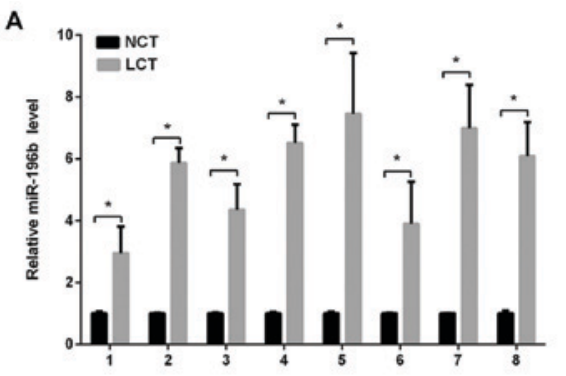

C

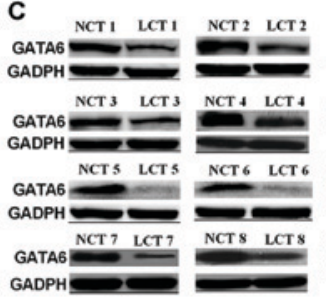

E

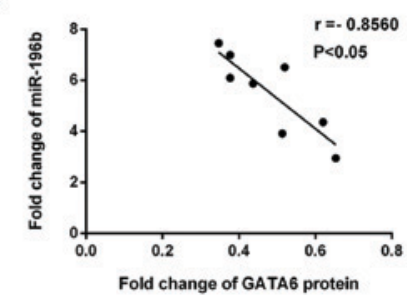

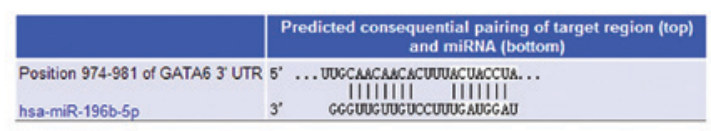

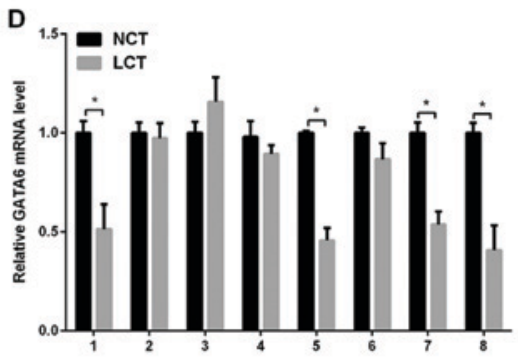

$\mathbf{F}$

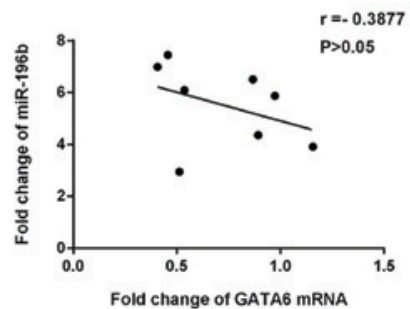

Figure 1. Detection of the miR-196b and GATA6 expressions in human LCT. (A) Analysis of the miR-196b expression in eight LCT and NCT by RT-qPCR. (B) Schematic diagram of the binding sites of miR-196b with the 3'-UTR of PDCD4. (C) Analysis of the GATA6 protein level in eight LCT and NCT tissues by western blot analysis. (D) The analysis of the GATA6 mRNA expression level in eight LCT and NCT tissues by RT-qPCR. (E) Regression analysis of the miR-196b mRNA expression and GATA6 protein in NSCLC tissues. (F) Regression analysis of the miR-196b and GATA6 mRNA expressions in NSCLC tissues. ${ }^{*} \mathrm{P}<0.05$. LCT, lung cancer tissues; NCT, non-cancerous tissues; NSCLC, non-small cell lung cancer.

A

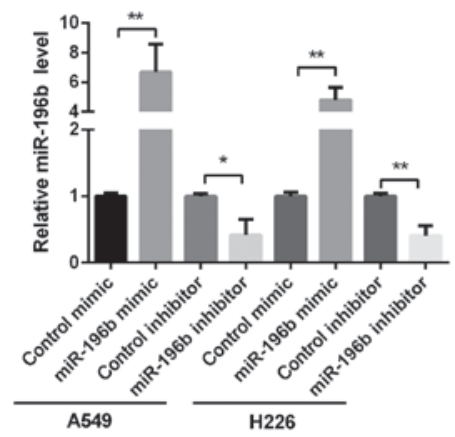

C

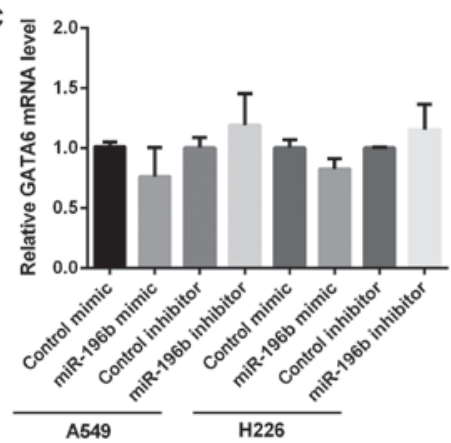

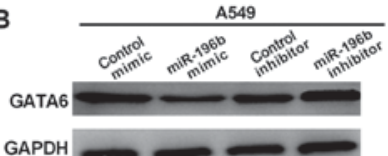
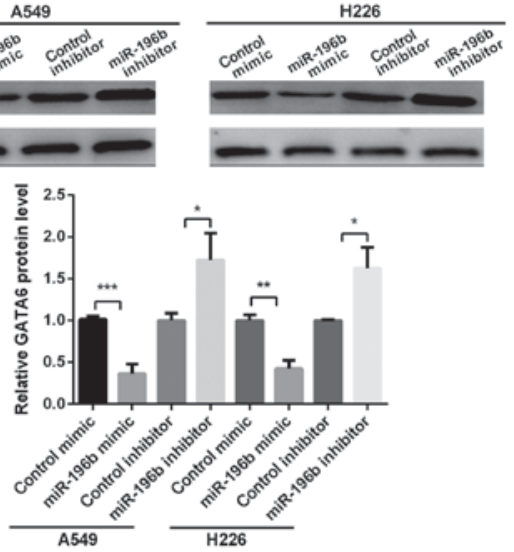

D

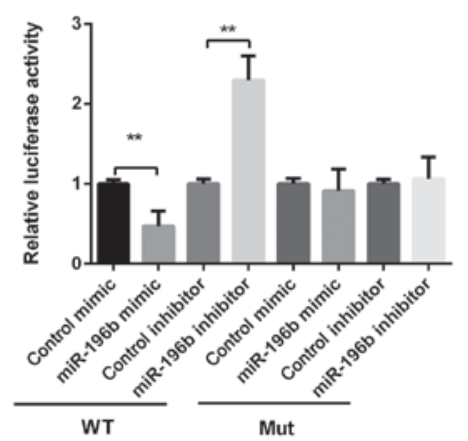

Figure 2. Regulation of GATA6 expression by miR-196b at post-transcriptional level. (A) Analysis of miR-196b expression in A549 and H226 cells after treatment with different transfection by RT-qPCR. (B) Analysis of GATA6 protein level in A549 and $\mathrm{H} 226$ cells after treatment with different transfection by western blotting. (C) The analysis of GATA6 mRNA in A549 and H226 cells after treatment with different transfection by RT-qPCR. (D) The relative luciferase activities in A549 cells after being transfected with the miR-196b mimic or inhibitor in wild-type or mutant type. ${ }^{*} \mathrm{P}<0.05 ;{ }^{* *} \mathrm{P}<0.01$. 


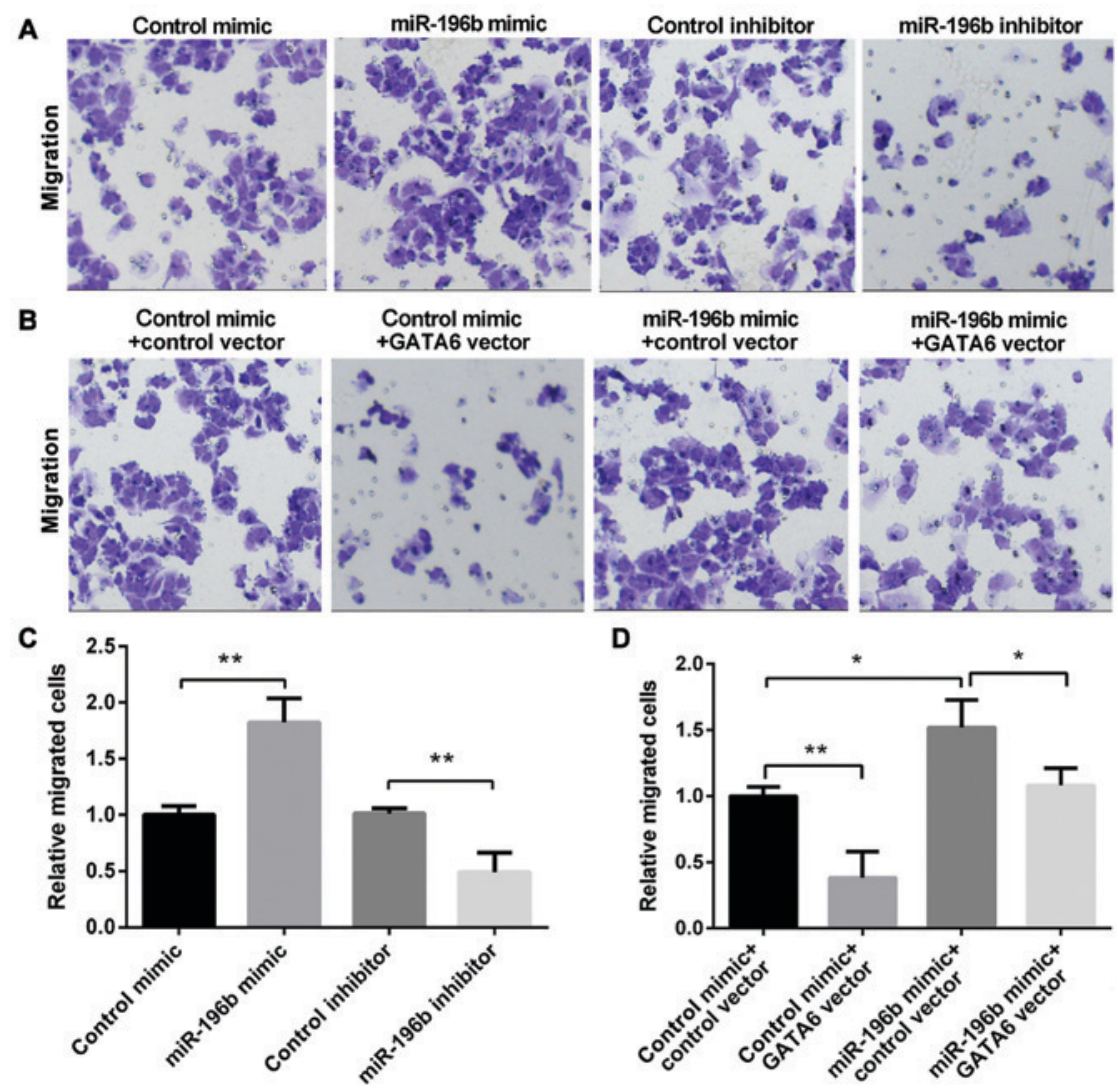

Figure 3. The effect of GATA6 on the migration of NSCLC regulated by miR-196b. (A-C) Detection and quantification of A549 cell migration after treatment with different transfection by Transwell migration assays. (B-D) Detection and quantification of $\mathrm{H} 226$ cell migration after treatment with different transfection by Transwell migration assays. NSCLC, non-small cell lung cancer. ${ }^{*} \mathrm{P}<0.05 ;{ }^{* *} \mathrm{P}<0.01$.

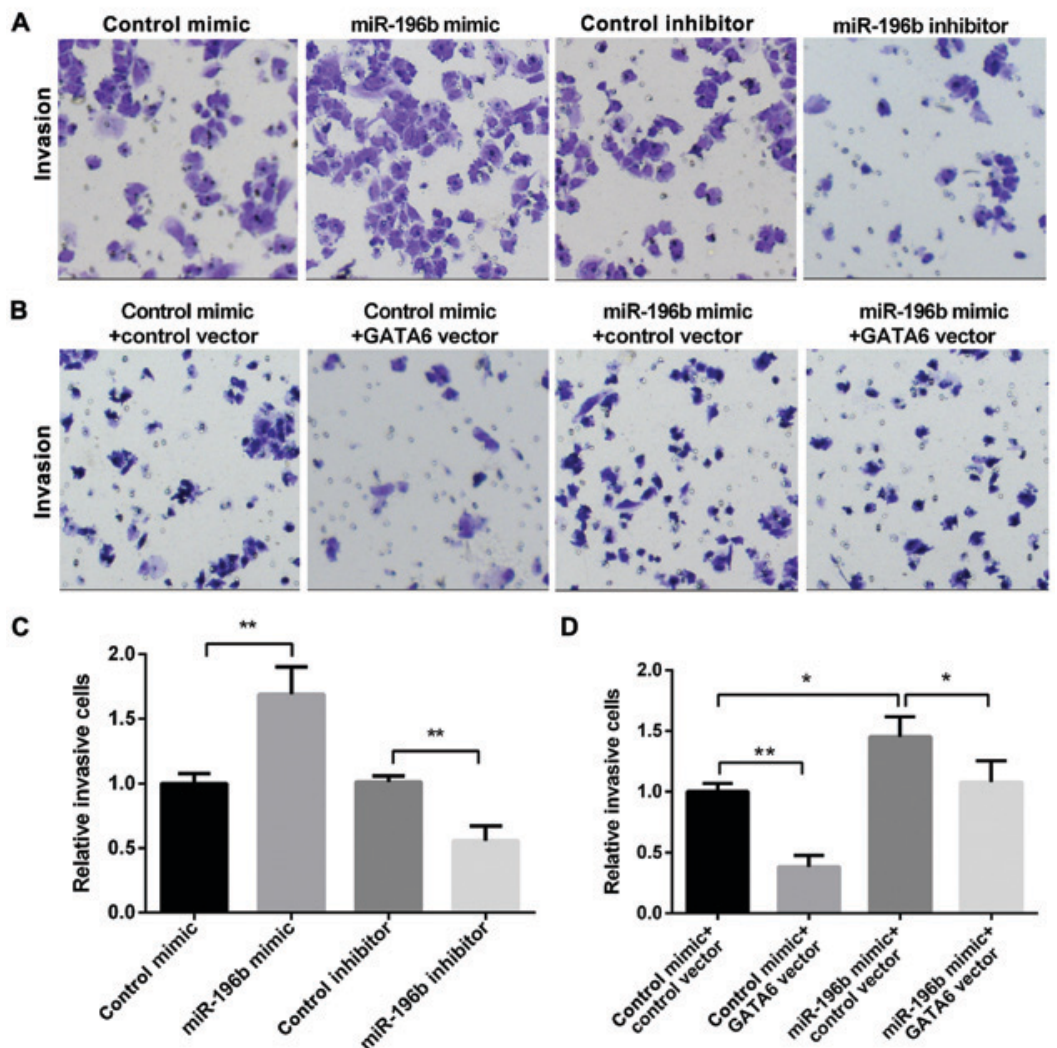

Figure 4. The effect of GATA6 on the invasion of NSCLC regulated by miR-196b. (A-C) Detection and quantification of A549 cell invasion after treatment with different transfection. (B-D) Detection and quantification of $\mathrm{H} 226$ cell invasion after treatment with different transfection. NSCLC, non-small cell lung cancer. ${ }^{*} \mathrm{P}<0.05 ;{ }^{* *} \mathrm{P}<0.01$. 
and miRanda to verify the direct target of miR-196b and it was found that GATA6 may be the target of miR-196b. The predicted target sites between miR-196b and GATA6 are shown in Fig. 1B. It is generally considered that the expression level of miRNAs and their target genes are opposite. Next, we investigated the GATA6 protein level in the same eight NSCLC and non-cancerous tissues, as shown in Fig. 1C. The GATA6 protein expression level was conspicuously lower in the NSCLC than in normal tissues, while the GATA6 mRNA level varied randomly (Fig. 1D). Regression analysis of correlation was used to show the relationship between the miR-196b and GATA6 protein levels (Fig. 1E) and miR-196b and GATA6 mRNA levels (Fig. 1F). We found that the inverse correlation coefficient $(r=-0.7394)$ between the miR-196b expression and GATA6 protein level was higher than that of the miR-196b expression and GATA6 mRNA level ( $\mathrm{r}=-0.3375)$. The results strongly suggested that miR-196b enhanced NSCLC cell migration and invasion by downregulating the GATA6 protein level and not the mRNA level.

Corroboration of GATA6 as a direct target of $\mathrm{miR}-196 \mathrm{~b}$. To further examine the relationship between miR-196b and GATA6, we evaluated the GATA6 expression in A549 and H226 cells after transfection with miR-196b mimic or inhibitor (Fig. 2A). It was shown that GATA6 protein level was markedly reduced after the overexpression of miR-196b but significantly increased when silencing miR-196b in A549 and H226 cells (Fig. 2B). However, the GATA6 mRNA level did not change for overexpressed or silenced miR-196b (Fig. 2C), suggesting that miR-196b regulated GATA6 expression at the post-transcriptional level.

We used dual luciferase reporter assay to detect the predicted sequence binding sites of miR-196b and GATA6. As shown in Fig. 2D, the luciferase reporter activity in the miR-196b mimic group was obviously lower, whereas the luciferase reporter activity in the miR-196b inhibitor group was obviously higher than that in the control group (Fig. 2D). Then, we detected miR-196b binding ability in the mutated type of miR-196b. The results showed that miR-196b mimic or miR-196b inhibitor group exert no effect on the luciferase reporter activity (Fig. 2D). Thus, miR-196b inhibited GATA6 translation by binding to the 3'-UTR of GATA6.

miR-196b promotes NSCLC migration by targeting GATA6. We used the Transwell assay to investigate the role that miR-196b and GATA6 played on NSCLC migration. The miR-196b mimic group showed an enhanced migration, whereas the miR-196b inhibitor group showed a decreased migration (Fig. 3A and B). Moreover, the overexpression of GATA6 decreased cell migration, while miR-196b mimic promoted cell migration. However, re-expression of both miR-196b and GATA6 showed lower migration than the cell overexpression of miR-196b alone (Fig. 3C and D), suggesting that GATA6 may attenuate the promotion effect of miR-196b on NSCLC migration. In conclusion, miR-196b may promote NSCLC cell migration by targeting GATA6.

miR-196b promotes invasion of NSCLC via targeting GATA6. Next, we used the Transwell assay to investigate the role that miR-196b and GATA6 played in NSCLC invasion.
The miR-196b mimic group showed increased invasion, while, the miR-196b inhibitor group showed decreased invasion (Fig. 4A and B). Moreover, the overexpression of GATA6 decreased cell invasion, while the miR-196b mimic promoted cell invasion. However, re-expression of both miR-196b and GATA6 showed lower invasion than that of the cell overexpression of miR-196b alone (Fig. 4C and D), suggesting that GATA6 may attenuate the promotion effect of miR-196b on NSCLC invasion. In conclusion, miR-196b may promote NSCLC cell invasion by targeting GATA6.

\section{Discussion}

It has been proven that miR-196 family members participate in tumorigenesis, and they are misexpressed in various malignancies $(20,21)$. For instance, miR-196b could promote tumor progression in lung cancers as an oncogene (22). In this study, we stated that miR-196b expression was markedly increased in NSCLC tissues and cell lines, which was consistent with a previous study where miR-196b was upregulated in the early-stage of NSCLC patients (23), and acted as a potential marker in lung cancer patients (12). We also confirmed that the miR-196b mimic may promote NSCLC cell migration and invasion, while the miR-196b inhibitor suppresses migration and invasion.

miRNAs regulated post-transcriptional gene expression by binding to the 3'UTR of target mRNAs (24). miR-196b was proven to regulate the GATA6 expression in colorectal cancer cells (25). However, our results have shown that GATA6 was regulated by miR-196b in NSCLC. miR-196b overexpression may inhibit the GATA6 expression in NSCLC cells, while the inhibition of miR-196b stimulated the GATA6 expression.

GATA6 is a member of the GATA family of Zn-finger transcription factors which are involved in the development of several tissues and organs $(15,26)$. A recent study suggests that modulating the expression of GATA6 may affect the growth of NSCLC cells (27). Mehta et al verified GATA6 and NKX2-1 as diagnostic biomarkers of non-invasive lung cancer (28). BMP4 regulation by GATA6 showed that it can inhibit tumorigenesis and metastasis of lung adenocarcinoma cells (29). In this study, we found that the GATA6 expression in NSCLC tumor was remarkably lower than that in the normal tissues. Pearson's correlation scatter plot showed that the relationship between the miR-196b expression and GATA6 was negatively correlated in NSCLC tissues. Furthermore, we confirmed that the overexpression of GATA6 may inhibit NSCLC cell migration and invasion. This is consistent with a previous study showing that GATA6 may inhibit lung adenocarcinoma metastasis (19). Additionally, we found that the overexpression of GATA6 may partially reverse the function of miR-196b.

In conclusion, we have demonstrated that miR-196b was markedlly increased and negatively associated with GATA6 in NSCLC cells. Moreover, the overexpression of miR-196b enhanced NSCLC cell migration and invasion via direct targeting GATA6. The results strongly suggest that GATA6 plays a significant role in regulating the progression of lung cancer.

\section{Acknowledgements}

Not applicable. 


\section{Funding}

No funding was received.

\section{Availability of data and materials}

The datasets used and/or analyzed during the present study are available from the corresponding author on reasonable request.

\section{Authors' contributions}

HL wrote the manuscript and collected tissue samples. CF was responsible for cell culture. SS performed PCR. All authors have read and approved the final study.

\section{Ethics approval and consent to participate}

The study was approved by the Ethics Committee of The Eastern Medical District of Linyi People's Hospital (Linyi, China) and all patients signed written informed consent after surgery.

\section{Consent for publication}

Not applicable.

\section{Competing interests}

The authors declare that they have no competing interests.

\section{References}

1. Torre LA, Bray F, Siegel RL, Ferlay J, Lortet-Tieulent J and Jemal A: Global cancer statistics, 2012. CA Cancer J Clin 65 : 87-108, 2015.

2. Jemal A, Bray F, Center MM, Ferlay J, Ward E and Forman D: Global cancer statistics. CA Cancer J Clin 61: 69-90, 2011.

3. Fassina A, Cappellesso R and Fassan M: Classification of non-small cell lung carcinoma in transthoracic needle specimens using microRNA expression profiling. Chest 140: 1305-1311, 2011

4. Yoo AS, Staahl BT, Chen L and Crabtree GR: MicroRNA-mediated switching of chromatin-remodelling complexes in neural development. Nature 460: 642-646, 2009.

5. Madhyastha R, Madhyastha H, Pengjam Y, Nakajima Y, Omura S and Maruyama M: NFkappaB activation is essential for miR-21 induction by TGF $\beta 1$ in high glucose conditions. Biochem Biophys Res Commun 451: 615-621, 2014.

6. Han H, Sun D, Li W, Shen H, Zhu Y, Li C, Chen Y, Lu L, Li W, Zhang J, et al: A c-Myc-MicroRNA functional feedback loop affects hepatocarcinogenesis. Hepatology 57: 2378-2389, 2013.

7. Mueller DW and Bosserhoff AK: MicroRNA miR-196a controls melanoma-associated genes by regulating $\mathrm{HOX}-\mathrm{C} 8$ expression. Int J Cancer 129: 1064-1074, 2011.

8. Schimanski CC, Frerichs K, Rahman F, Berger M, Lang H, Galle PR, Moehler M and Gockel I: High miR-196a levels promote the oncogenic phenotype of colorectal cancer cells World J Gastroenterol 15: 2089-2096, 2009.

9. Popovic R, Riesbeck LE, Velu CS, Chaubey A, Zhang J, Achille NJ, Erfurth FE, Eaton K, Lu J, Grimes HL, et al: Regulation of mir-196b by MLL and its overexpression by MLL fusions contributes to immortalization. Blood 113: 3314-3322, 2009.

10. Guan Y, Mizoguchi M, Yoshimoto K, Hata N, Shono T, Suzuki SO, Araki Y, Kuga D, Nakamizo A, Amano T, et al: miRNA-196 is upregulated in glioblastoma but not in anaplastic astrocytoma and has prognostic significance. Clin Cancer Res 16: 4289-4297, 2010

11. Li Y, Zhang M, Chen H, Dong Z, Ganapathy V, Thangaraju M and Huang S: Ratio of miR-196s to HOXC8 messenger RNA correlates with breast cancer cell migration and metastasis. Cancer Res 70: 7894-7904, 2010.
12. Li X, Shi Y, Yin Z, Xue X and Zhou B: An eight-miRNA signature as a potential biomarker for predicting survival in lung adenocarcinoma. J Transl Med 12: 159, 2014.

13. Zheng R and Blobel GA: GATA transcription factors and cancer. Genes Cancer 1: 1178-1188, 2010.

14. Aronson BE, Stapleton KA and Krasinski SD: Role of GATA factors in development, differentiation, and homeostasis of the small intestinal epithelium. Am J Physiol Gastrointest Liver Physiol 306: G474-G490, 2014

15. Tian F, Li D, Chen J, Liu W, Cai L, Li J, Jiang P, Liu Z, Zhao X, Guo F, et al: Aberrant expression of GATA binding protein 6 correlates with poor prognosis and promotes metastasis in cholangiocarcinoma. Eur J Cancer 49: 1771-1780, 2013.

16. Tian F, Chen J, Zheng S, Li D, Zhao X, Jiang P, Li J and Wang S: miR-124 targets GATA6 to suppress cholangiocarcinoma cell invasion and metastasis. BMC Cancer 17: 175, 2017.

17. Haveri H, Westerholm-Ormio M, Lindfors K, Mäki M, Savilahti E, Andersson LC and Heikinheimo M: Transcription factors GATA-4 and GATA-6 in normal and neoplastic human gastrointestinal mucosa. BMC Gastroenterol 8: 9, 2008.

18. Belaguli NS, Aftab M, Rigi M, Zhang M, Albo D and Berger DH: GATA6 promotes colon cancer cell invasion by regulating urokinase plasminogen activator gene expression. Neoplasia 12: 856-865, 2010

19. Cheung WK, Zhao M, Liu Z, Stevens LE, Cao PD, Fang JE, Westbrook TF and Nguyen DX: Control of alveolar differentiation by the lineage transcription factors GATA6 and HOPX inhibits lung adenocarcinoma metastasis. Cancer Cell 23: 725-738, 2013.

20. Chen C, Zhang Y, Zhang L, Weakley SM and Yao Q: MicroRNA-196: Critical roles and clinical applications in development and cancer. J Cell Mol Med 15: 14-23, 2011.

21. Lu YC, Chang JT, Liao CT, Kang CJ, Huang SF, Chen IH, Huang CC, Huang YC, Chen WH, Tsai CY, et al: OncomiR-196 promotes an invasive phenotype in oral cancer through the NME4-JNK-TIMP1-MMP signaling pathway. Mol Cancer 13: 218,2014

22. Hamamoto J, Soejima K, Yoda S, Naoki K, Nakayama S, Satomi R, Terai H, Ikemura S, Sato T, Yasuda H, et al: Identification of microRNAs differentially expressed between lung squamous cell carcinoma and lung adenocarcinoma. Mol Med Rep 8: 456-462, 2013.

23. Võsa U, Vooder T, Kolde R, Fischer K, Välk K, Tõnisson N, Roosipuu R, Vilo J, Metspalu A and Annilo T: Identification of miR-374a as a prognostic marker for survival in patients with early-stage nonsmall cell lung cancer. Genes Chromosomes Cancer 50: 812-822, 2011.

24. Chen K and Rajewsky N: The evolution of gene regulation by transcription factors and microRNAs. Nat Rev Genet 8: 93-103, 2007.

25. Fantini S, Salsi V, Reggiani L, Maiorana A and Zappavigna V: The miR-196b miRNA inhibits the GATA6 intestinal transcription factor and is upregulated in colon cancer patients. Oncotarget 8: 4747-4759, 2017.

26. Kwei KA, Bashyam MD, Kao J, Ratheesh R, Reddy EC, Kim YH, Montgomery K, Giacomini CP, Choi Y-L, Chatterjee S, et al: Genomic profiling identifies GATA6 as a candidate oncogene amplified in pancreatobiliary cancer. PLoS Genet 4: e1000081, 2008.

27. Zito G, Naselli F, Saieva L, Raimondo S, Calabrese G, Guzzardo C, Forte S, Rolfo C, Parenti R and Alessandro R: Retinoic acid affects lung adenocarcinoma growth by inducing differentiation via GATA6 activation and EGFR and Wnt inhibition. Sci Rep 7: 4770, 2017.

28. Mehta A, Cordero J, Dobersch S, Romero-Olmedo AJ, Savai R, Bodner J, Chao CM, Fink L, Guzmán-Díaz E, Singh I, et al: Non-invasive lung cancer diagnosis by detection of GATA6 and NKX2-1 isoforms in exhaled breath condensate. EMBO Mol Med 8: 1380-1389, 2016

29. Kim JS, Kurie JM and Ahn YH: BMP4 depletion by miR-200 inhibits tumorigenesis and metastasis of lung adenocarcinoma cells. Mol Cancer 14: 173, 2015.

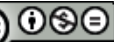

This work is licensed under a Creative Commons Attribution-NonCommercial-NoDerivatives 4.0 International (CC BY-NC-ND 4.0) License. 